\title{
SOME EXACT INEQUALITIES OF HARDY-LITTLEWOOD-POLYA TYPE FOR PERIODIC FUNCTIONS
}

\author{
LAITH EMIL AZAR
}

Received 28 September 2003

We investigate the following problem: for a given $A \geq 0$, find the infimum of the set of $B \geq 0$ such that the inequality $\left\|x^{(k)}\right\|_{2}^{2} \leq A\left\|x^{(r)}\right\|_{2}^{2}+B\|x\|_{2}^{2}$, for $k, r \in \mathbb{N} \cup\{0\}, 0 \leq k<r$, holds for all sufficiently smooth functions.

2000 Mathematics Subject Classification: 41A17, 42A05.

1. Introduction. Let $G=\mathbb{R}$ or $G=\mathbb{T}=[0,2 \pi)$. By $L_{2}(G)$, we will denote the spaces of all measurable functions $x: G \rightarrow \mathbb{R}$ such that

$$
\|x\|_{2}=\|x\|_{L_{2}(G)}:=\left\{\int_{G}|x(t)|^{2} d t\right\}^{1 / 2}<\infty .
$$

Denote by $L_{2}^{r}(G)(r \in \mathbb{N})$ the space of all functions $x$ such that $x^{(r-1)}$ are locally absolutely continuous and $x^{(r)} \in L_{2}(G)$, and set $L_{2,2}^{r}(G)=L_{2}(G) \cap L_{2}^{r}(G)$ (in the case $G=\mathbb{T}$, we mean that spaces $L_{2}(G)$ and $L_{2}^{r}(G)$ consist of $2 \pi$-periodic functions). Note that $L_{2}^{r}(G) \subset L_{2}(G)$ if $G=\mathbb{T}$.

It is well known that the exact inequality of Hardy [3]

$$
\left\|x^{(k)}\right\|_{2}^{2} \leq\|x\|_{2}^{2(1-k / r)}\left\|x^{(r)}\right\|_{2}^{2(k / r)}, \quad k \in \mathbb{N}, 0<k<r,
$$

holds for every function $x \in L_{2,2}^{r}(\mathbb{R})$.

For any $A>0$ and any $x \in L_{2,2}^{r}(\mathbb{R})$, from inequality (1.2), we get

$$
\left\|x^{(k)}\right\|_{2}^{2} \leq\left\{\left(\frac{k}{A r}\right)^{k /(r-k)}\|x\|_{2}^{2}\right\}^{(r-k) / r}\left\{\frac{A r}{k}\left\|x^{(r)}\right\|_{2}^{2}\right\}^{k / r}
$$

Using Young's inequality

$$
a b \leq \frac{a^{p}}{p}+\frac{b^{p^{\prime}}}{p^{\prime}}, \quad \frac{1}{p}+\frac{1}{p^{\prime}}=1, \quad 1 \leq p<\infty, a, b>0,
$$

with $p=r /(r-k)$ and $p^{\prime}=r / k$, we get, for any $A>0$ and any $x \in L_{2,2}^{r}(\mathbb{R})$, the following inequality:

$$
\left\|x^{(k)}\right\|_{2}^{2} \leq A\left\|x^{(r)}\right\|_{2}^{2}+\frac{r-k}{r}\left(\frac{k}{A r}\right)^{k /(r-k)}\|x\|_{2}^{2}
$$


This inequality is the best possible in the next sense: for a given $A>0$, the infimum of constants $B$ such that the inequality

$$
\left\|x^{(k)}\right\|_{2}^{2} \leq A\left\|x^{(r)}\right\|_{2}^{2}+B\|x\|_{2}^{2}
$$

holds for all functions $x \in L_{2,2}^{r}(\mathbb{R})$ is equal to

$$
\frac{r-k}{r}\left(\frac{k}{A r}\right)^{k /(r-k)}
$$

As is well known, inequality (1.2) (and consequently (1.5)) holds true for any function $x \in L_{2,2}^{r}(\mathbb{T})$. However, the constant (1.7) is not the best possible in general (for a given constant $A$ ). Therefore, the main problem which we will study in this paper is the following.

For a given $A \geq 0$, find the infimum of constants $B$ such that inequality (1.6) holds for all functions $x \in L_{2,2}^{r}(\mathbb{T})$.

We will denote this infimum by $\Psi(\mathbb{T} ; r, k ; A)$. We will investigate also the analogous problem in the presence of some restrictions on the spectrum of functions $x \in L_{2,2}^{r}(\mathbb{T})$.

Note that Babenko and Rassias [1] investigated the problem on exact inequalities for functions $x \in L_{2,2}^{r}(\mathbb{T})$. They have found, for a given $A \geq 0$, the infimum of constants $B$ such that the inequality

$$
\left\|x^{(k)}\right\|_{2}^{2} \leq A\|x\|_{2}^{2}+B\left\|x^{(r)}\right\|_{2}^{2}
$$

holds for all functions $x \in L_{2,2}^{r}(\mathbb{T})$.

For more information related to this subject, see, for example, [2, 4, 5, 6].

\section{Main results}

THEOREM 2.1. Let $k, r \in \mathbb{N}, k<r$. Then for any $A \geq 0$ and any $x \in L_{2,2}^{r}(\mathbb{T})$,

$$
\left\|x^{(k)}\right\|_{2}^{2} \leq A\left\|x^{(r)}\right\|_{2}^{2}+\left(v_{0}^{2 k}-A v_{0}^{2 r}\right)\|x\|_{2}^{2}=A\left\|x^{(r)}\right\|_{2}^{2}+\varphi\left(A, v_{0}\right)\|x\|_{2}^{2}
$$

holds if $v_{0}$ is such that $\eta\left(v_{0}+1\right) \leq A \leq \eta\left(v_{0}\right)$, where

$$
\eta(v)=\frac{v^{2 k}-(v-1)^{2 k}}{v^{2 r}-(v-1)^{2 r}} .
$$

Given $A$, the constant $\varphi\left(A, v_{0}\right)$ in (2.1) is the best possible; that is,

$$
\Psi(\mathbb{T} ; r, k ; A)=\left(v_{0}^{2 k}-A v_{0}^{2 r}\right),
$$

where $v_{0}$ is such that $\eta\left(v_{0}+1\right) \leq A \leq \eta\left(v_{0}\right)$.

Proof. Let

$$
\begin{aligned}
& e_{v}(t):=\frac{1}{2 \pi} e^{i v t}, \quad v \in \mathbb{Z}, t \in \mathbb{R}, \\
& c_{v}(x)=\int_{0}^{2 \pi} x(t) e_{v}(t) d t
\end{aligned}
$$


be Fourier coefficients of a function $x$, and let

$$
\sum_{v \in \mathbb{Z}} c_{v}(x) e_{v}(t)
$$

be the Fourier series of a function $x$.

For any $x \in L_{2,2}^{r}(\mathbb{T}), 0<k<r$, and any $A \geq 0$, using Parseval's equality, we get

$$
\begin{aligned}
\left\|x^{(k)}\right\|_{2}^{2} & =\sum_{\substack{v \in \mathbb{Z} \\
v \neq 0}}\left|c_{v}(x)\right|^{2} v^{2 k} \\
& =A \sum_{\substack{v \in \mathbb{Z} \\
v \neq 0}}\left|c_{v}(x)\right|^{2} v^{2 r}+\sum_{\substack{v \in \mathbb{Z} \\
v \neq 0}}\left|c_{v}(x)\right|^{2} v^{2 r}\left[\frac{v^{2 k}}{v^{2 r}}-A\right] \\
& =A\left\|x^{(r)}\right\|_{2}^{2}+\sum_{\substack{v \in \mathbb{Z} \\
v \neq 0}}\left|c_{v}(x)\right|^{2}\left[v^{2 k}-A v^{2 r}\right] \\
& \leq A\left\|x^{(r)}\right\|_{2}^{2}+\max _{v \in \mathbb{N}}\left[v^{2 k}-A v^{2 r}\right] \sum_{\substack{v \in \mathbb{Z} \\
v \neq 0}}\left|c_{v}(x)\right|^{2} \\
& =A\left\|x^{(r)}\right\|_{2}^{2}+\max _{v \in \mathbb{N}}\left[v^{2 k}-A v^{2 r}\right]\|x\|_{2}^{2} .
\end{aligned}
$$

Set

$$
\varphi(A, v):=v^{2 k}-A v^{2 r}
$$

then the last inequality can be written in the form

$$
\left\|x^{(k)}\right\|_{2}^{2} \leq A\left\|x^{(r)}\right\|_{2}^{2}+\max _{v \in \mathbb{N}} \varphi(A, v)\|x\|_{2}^{2} .
$$

Our goal now is to find for a given $A \geq 0$ the value of

$$
\max _{v \in \mathbb{N}} \varphi(A, v)
$$

We consider the difference

$$
\begin{aligned}
\delta_{v} & =\varphi(A, v)-\varphi(A, v-1) \\
& =v^{2 k}-A v^{2 r}-(v-1)^{2 k}+A(v-1)^{2 r} \\
& =A\left[(v-1)^{2 r}-v^{2 r}\right]-\left[(v-1)^{2 k}-v^{2 k}\right] \\
& =\left[(v)^{2 r}-(v-1)^{2 r}\right]\left[\frac{v^{2 k}-(v-1)^{2 k}}{v^{2 r}-(v-1)^{2 r}}-A\right] .
\end{aligned}
$$

Set, for $v \in \mathbb{N}$,

$$
\eta(v):=\frac{v^{2 k}-(v-1)^{2 k}}{v^{2 r}-(v-1)^{2 r}}
$$

then the last equality can be written in the form

$$
\delta_{v}=\left[(v)^{2 r}-(v-1)^{2 r}\right][\eta(v)-A] .
$$


It is not difficult to see that

$$
\operatorname{sgn} \delta_{v}=\operatorname{sgn}[\eta(v)-A] .
$$

We now study the function $\eta(v)$.

Note that $\eta(1)=1, \eta(v) \rightarrow 0$ as $v \rightarrow \infty$ (since $k<r$ ), and, for $v \geq 1$,

$$
\eta(v)>\eta(v+1)
$$

Indeed, using Cauchy's theorem,

$$
\eta(v)=\frac{k}{r} \frac{\theta_{v}^{2 k}}{\theta_{v}^{2 r}}, \quad v-1<\theta_{v}<v
$$

Thus, inequality (2.14) is equivalent to the inequality

$$
\frac{k}{r} \frac{\theta_{v}^{2 k}}{\theta_{v}^{2 r}}>\frac{k}{r} \frac{\theta_{v+1}^{2 k}}{\theta_{v+1}^{2 r}}
$$

or

$$
\left(\frac{\theta_{v}}{\theta_{v+1}}\right)^{2 r-2 k}<1 .
$$

The last inequality is true since $\theta_{v}<\theta_{v+1}$ and $2 r-2 k>0$.

If, for a given $A \geq 0$, the value $v_{0}$ is such that $\eta\left(v_{0}+1\right) \leq A \leq \eta\left(v_{0}\right)$, then for $v \leq v_{0}$, taking into account equality (2.13), we obtain that $\delta_{v} \geq 0$, and consequently,

$$
\varphi(A, 1) \leq \varphi(A, 2) \leq \cdots \leq \varphi\left(A, v_{0}\right) .
$$

In the case $v>v_{0}$, we get $\delta_{v} \leq 0$ and then

$$
\varphi\left(A, v_{0}\right) \geq \varphi\left(A, v_{0}+1\right) \geq \cdots .
$$

Therefore,

$$
\max _{v \in \mathbb{N}} \varphi(A, v)=\max _{v \in \mathbb{N}}\left[v^{2 k}-A v^{2 r}\right]=\varphi\left(A, v_{0}\right)
$$

if $\eta\left(v_{0}+1\right) \leq A \leq \eta\left(v_{0}\right)$. Thus inequality (2.1) is proved.

We now show the evidence of equality (2.3). Let $x(t)=\cos v_{0} t$. Then the inequality becomes an equality since

$$
\left\|x^{(k)}\right\|_{2}^{2}=\pi v_{0}^{2 k}, \quad\|x\|_{2}^{2}=\pi, \quad\left\|x^{(r)}\right\|_{2}^{2}=\pi v_{0}^{2 r} .
$$

The function $\Psi(\mathbb{T} ; r, k ; A)$ defined by (2.3) is continuous, linear on any interval $[\eta(v+$ $1), \eta(v)]$, and for any $v \geq 1$,

$$
\Psi(\mathbb{T} ; r, k ; \eta(v+1))=\frac{v^{2 k}(v+1)^{2 r}-v^{2 r}(v+1)^{2 k}}{(v+1)^{2 r}-v^{2 r}} .
$$


Claim that

$$
v_{0}^{2 k}-A v_{0}^{2 r}<\frac{r-k}{r}\left(\frac{k}{A r}\right)^{k /(r-k)}
$$

To do this, we will consider the function

$$
f(A)=\frac{r-k}{r}\left(\frac{k}{A r}\right)^{k /(r-k)}-v^{2 k}+A v^{2 r} .
$$

Differentiating the function $f$, we get

$$
f^{\prime}(A)=v^{2 r}-\left(\frac{k}{r}\right)^{r /(r-k)}\left(\frac{1}{A}\right)^{r /(r-k)}
$$

and the condition $f^{\prime}(A)=0$ implies

$$
A_{0}=\frac{k}{r} v^{2 k-2 r}
$$

Now we have $f\left(A_{0}\right)=0$ and our statement is proved.

Let $\Pi_{2 n+1}$ be the set of trigonometric polynomials of order less than or equal to $n$. Then in view of the Bernstein-type inequality, we have, for any $\tau \in \Pi_{2 n+1}$ and any $k \in \mathbb{N}$,

$$
\left\|\tau^{(k)}\right\|_{2}^{2} \leq n^{2 k}\|\tau\|_{2}^{2}
$$

Therefore, for $x=\tau$, inequality (1.6) holds with $A=0$ and $B=n^{2 k}$. Let now $A>0$. By repeating (with obvious modifications) the proof of Theorem 2.1, we obtain that for any $k, r \in \mathbb{N}, k<r$, and any $\tau \in \Pi_{2 n+1}$, the following holds:

$$
\left\|\boldsymbol{\tau}^{(k)}\right\|_{2}^{2} \leq A\left\|\boldsymbol{\tau}^{(r)}\right\|_{2}^{2}+B\|\boldsymbol{\tau}\|_{2}^{2}=A\left\|\boldsymbol{\tau}^{(r)}\right\|_{2}^{2}+\max _{\substack{v \in \mathbb{N} \\ v \leq n}} \varphi(A, v)\|\boldsymbol{\tau}\|_{2}^{2} .
$$

We now compute the value

$$
\max _{\substack{v \in \mathbb{N} \\ v \leq n}} \varphi(A, v)
$$

Let $\eta\left(v_{0}+1\right) \leq A \leq \eta\left(v_{0}\right)$, where $v_{0} \leq n$. Then

$$
\max _{\substack{v \in \mathbb{N} \\ v \leq n}} \varphi(A, v)=\varphi\left(A, v_{0}\right)=\max _{v \in \mathbb{N}} \varphi(A, v)
$$

If $\eta\left(v_{0}+1\right) \leq A \leq \eta\left(v_{0}\right)$, where $v_{0} \geq n+1$, we get, taking into account the relations

$$
\varphi(A, 1) \leq \varphi(A, 2) \leq \cdots \leq \varphi(A, n) \leq \cdots \leq \varphi\left(A, v_{0}\right)
$$

that

$$
\max _{\substack{v \in \mathbb{N} \\ v \leq n}} \varphi(A, v)=\varphi(A, n)=n^{2 k}-A n^{2 r}
$$

if $A \leq \eta(n)$. Therefore, we have proved the following theorem. 
THEOREM 2.2. For any $k, n, r \in \mathbb{N}, k<r$, any $\tau \in \Pi_{2 n+1}$, and any $A \geq 0$,

$$
\left\|\boldsymbol{\tau}^{(k)}\right\|_{2}^{2} \leq A\left\|\boldsymbol{\tau}^{(r)}\right\|_{2}^{2}+B\|\tau\|_{2}^{2},
$$

where

$$
B=\varphi\left(A, v_{0}\right)
$$

if $\eta\left(v_{0}+1\right) \leq A \leq \eta\left(v_{0}\right), v_{0} \leq n$, and

$$
B=\varphi(A, n)
$$

if $A \leq \eta(n)$. Inequality (2.33) is the best possible for any $A \geq 0$.

Consider the set of functions $x \in L_{2,2}^{r}(\mathbb{T})$ such that $c_{v}(x)=0$ for $|v| \leq n-1$ (we will denote this set of functions by $L_{2,2}^{r}(\mathbb{T} ; n)$ ). The following inequality is well known for functions $x \in L_{2,2}^{r}(\mathbb{T} ; n)$ :

$$
\|x\|_{2}^{2} \leq \frac{1}{n^{2 r}}\left\|x^{(r)}\right\|_{2}^{2}
$$

Thus, for any $k<r$,

$$
\left\|x^{(k)}\right\|_{2}^{2} \leq \frac{1}{n^{2 r-2 k}}\left\|x^{(r)}\right\|_{2}^{2}
$$

Then inequality (1.6) for functions $x \in L_{2,2}^{r}$ ( $\mathbb{T} ; n$ ) holds with $B=0$ and $A \geq 1 / n^{2 r-2 k}$.

By repeating (with obvious modifications) the proof of Theorem 2.1, we obtain that for any $k, r \in \mathbb{N}, k<r$, any $x \in L_{2,2}^{r}(\mathbb{T} ; n)$, and any $0 \leq A \leq 1 / n^{2 r-2 k}$,

$$
\left\|x^{(k)}\right\|_{2}^{2} \leq A\left\|x^{(r)}\right\|_{2}^{2}+\max _{\substack{v \in \mathbb{N} \\ v \geq n}} \varphi(A, v)\|x\|_{2}^{2} .
$$

We need to find the value of

$$
\max _{\substack{v \in \mathbb{N} \\ v \geq n}} \varphi(A, v)
$$

Note that

$$
\eta(n)=\frac{n^{2 k}-(n-1)^{2 k}}{n^{2 r}-(n-1)^{2 r}} \leq \frac{n^{2 k}}{n^{2 r}}
$$

To show this, assume that

$$
\eta(n)>\frac{n^{2 k}}{n^{2 r}}
$$

then we get

$$
\left(\frac{n}{n-1}\right)^{2 r}<\left(\frac{n}{n-1}\right)^{2 k}
$$

which is impossible since $n /(n-1)>1$ and $k<r$. 
First let $\eta\left(v_{0}+1\right) \leq A \leq \eta\left(v_{0}\right)$ where $v_{0} \leq n$. Then

$$
\varphi(A, n) \geq \varphi(A, n+1) \geq \cdots
$$

and therefore

$$
\max _{\substack{v \in \mathbb{N} \\ v \geq n}} \varphi(A, v)=\varphi(A, n)
$$

if $\eta\left(v_{0}+1\right) \leq A \leq n^{2 k-2 r}$.

Let now $\eta\left(v_{0}+1\right) \leq A \leq \eta\left(v_{0}\right)$ where $v_{0} \geq n+1$. In this case, we get

$$
\max _{\substack{v \in \mathbb{N} \\ v \geq n}} \varphi(A, v)=\max _{v \in \mathbb{N}} \varphi(A, v)=\varphi\left(A, v_{0}\right)
$$

Thus we have proved the following theorem.

THEOREM 2.3. For any $k, n, r \in \mathbb{N}, k<r$, any $x \in L_{2,2}^{r}(\mathbb{T} ; n)$, and any $0 \leq A \leq n^{2 k-2 r}$, inequality (1.6) holds where $B=\varphi(A, n)$ if $\eta\left(v_{0}+1\right) \leq A \leq n^{2 k-2 r}, v_{0} \leq n$, and $B=$ $\varphi\left(A, v_{0}\right)$ if $\eta\left(v_{0}+1\right) \leq A \leq \eta\left(v_{0}\right), v_{0} \geq n+1$. Inequality (1.6) is the best possible for any $0 \leq A \leq n^{2 k-2 r}$.

\section{REFERENCES}

[1] V. F. Babenko and T. M. Rassias, On exact inequalities of Hardy-Littlewood-Polya type, J. Math. Anal. Appl. 245 (2000), no. 2, 570-593.

[2] B. D. Bojanov and A. K. Varma, On a polynomial inequality of Kolmogoroff's type, Proc. Amer. Math. Soc. 124 (1996), no. 2, 491-496.

[3] G. H. Hardy, J. E. Littlewood, and G. Polya, Inequalities, Cambridge University Press, Cambridge, 1934.

[4] N. P. Korneǐchuk, Exact Constants in Approximation Theory, Encyclopedia of Mathematics and Its Applications, vol. 38, Cambridge University Press, Cambridge, 1991.

[5] S. Z. Rafal'son, An inequality between norms of a function and its derivative in integral metrics, Mat. Zametki 33 (1983), no. 1, 77-82.

[6] A. K. Varma, A new characterization of Hermite polynomials, Acta Math. Hungar. 49 (1987), no. 1-2, 169-172.

Laith Emil Azar: Department of Mathematics, Al Al-Bayt University, Mafraq 25113, Jordan

E-mail address: azar_1aith@yahoo.com 


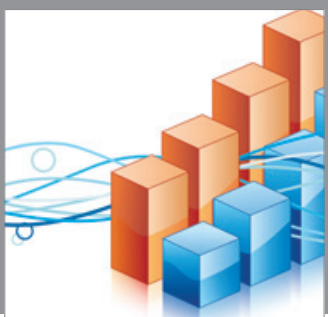

Advances in

Operations Research

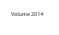

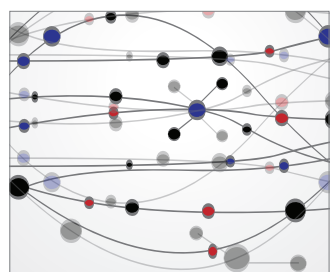

\section{The Scientific} World Journal
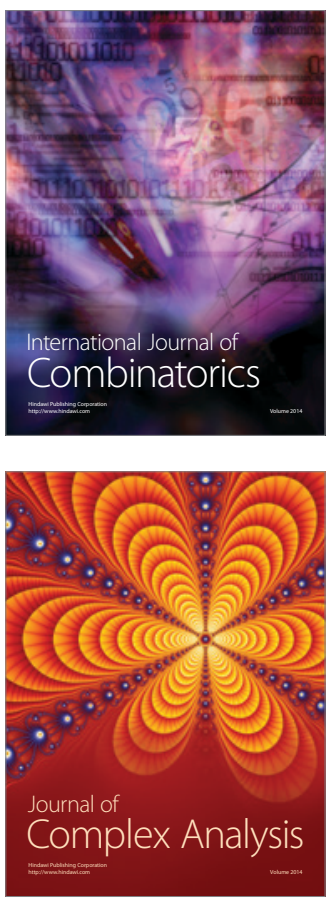

International Journal of

Mathematics and

Mathematical

Sciences
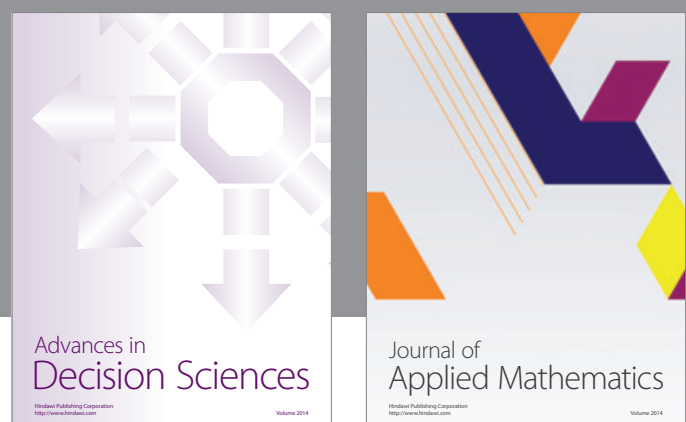

Journal of

Applied Mathematics
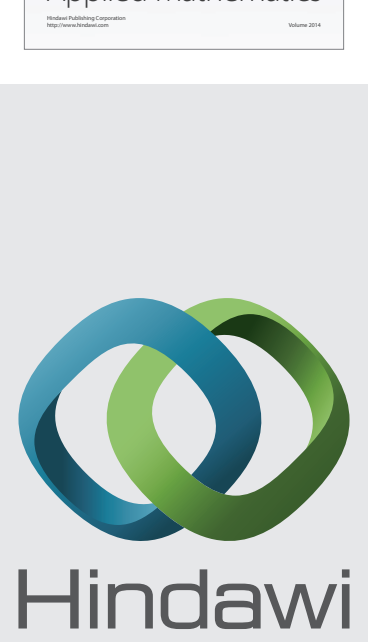

Submit your manuscripts at http://www.hindawi.com
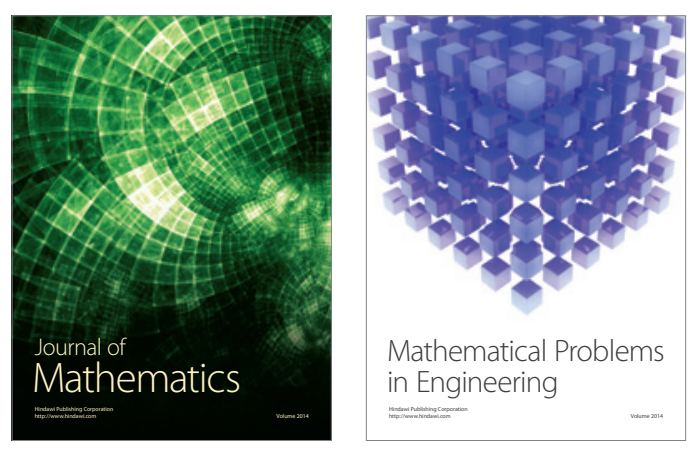

Mathematical Problems in Engineering
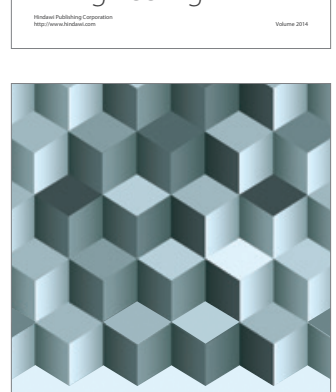

Journal of

Function Spaces
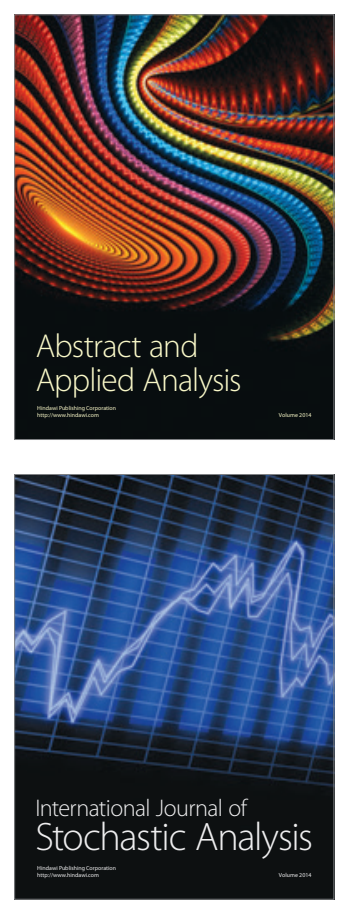

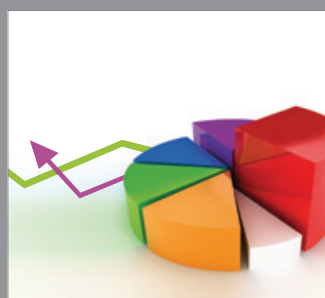

ournal of

Probability and Statistics

Promensencen
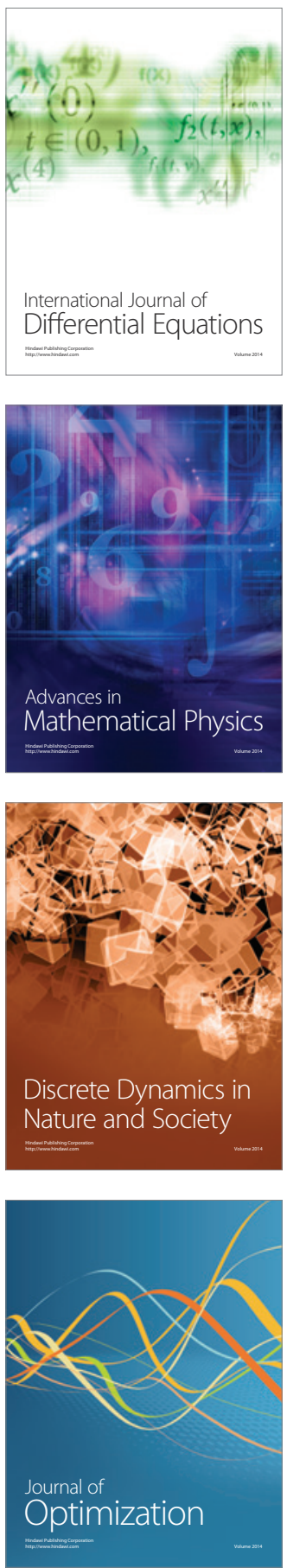